Cases: These cases comprised 6 patients examined at our hospital between April 1992 and November 2001. Past operations were keratoplasty in 5 eyes and cataract surgery in 1 eye. Clinical findings and predisposing factors were evaluated retrospectively. The period between herpetic epithelial keratitis onset and ophthalmic surgery ranged from 1.5 to 79 months. Predisposing factors included corticosteroid therapy and operative wound. The herpetic epithelial lesions were dendritic ulcers in 2 eyes, geographic ulcer in 1 eye, and atypical epithelial lesions in 3 eyes; in all cases, herpes simplex virus (HSV)-DNA was detected by polymerase chain reaction (PCR) in tear fluid. All herpetic epithelial lesions healed with oral and topical acyclovir.

Conclusions: When corticosteroids are used following ophthalmic surgery, physicians should be alert to the possibility of herpetic epithelial keratitis, even in patients with no clinical history of herpes simplex keratitis. PCR detection in tear fluid is helpful in diagnosing this disease.

Nippon Ganka Gakkai Zasshi (J Jpn Ophthalmol Soc 107:538-542, 2003)

Seiya Miyajima, Yoichiro Sano, Chie Sotozono, Norihiko Yokoi, Yutaka Ishino and Shigeru Kinoshita

Department of Ophthalmology, Kyoto Prefectural University of Medicine

DOI 10.1007/s10384-004-1015-5

\section{The Inhibitory Effect of Aldose Reductase Inhibitor on Proliferation in the Lens Epithelial Cells of Rat Sugar Cataract}

Purpose: To evaluate the inhibitory effect of aldose reductase inhibitor (ARI) on the accumulation of sugar alcohol and the enhanced proliferation of lens epithelial cells of rats fed a galactose diet.

Methods: Sprague-Dawley rats were fed a diet containing $50 \%$ galactose with or without ARI (SNK-860). Histological changes in the lenses were observed by light microscopy, and the amount of galactitol accumulated in the lens epithelium was measured by liquid-gas chromatography. Immunohistochemical staining of proliferating cell nuclear antigen (PCNA) in whole-mount preparations was performed to assay the proliferative ability of the lens epithelial cells.

Results: The amount of galactitol in the lens epithelium and the number of PCNA positive cells in rats administered ARI were less than in rats fed the galactose diet. Multilayered epithelium was observed in advanced cataract of rats fed the galactose diet, but not in the rats given ARI.

Conclusion: The administration of ARI can prevent cataractous changes and aberrant proliferation of lens epithelial cells.

Nippon Ganka Gakkai Zasshi (J Jpn Ophthalmol Soc 107:565-570, 2003)
Soichi Morikubo, Yoshihiro Takamura, Eri Kubo, Shosai Tsuzuki and Yoshio Akagi

Department of Ophthalmology, Fukui Medical University

DOI 10.1007/s10384-004-1016-4

\section{Localization of Glucose and Sorbitol in Incubated Bovine Lenses Using Chemical Shift Imaging}

Purpose: To use chemical shift imaging (CSI) to measure the metabolic changes in glucose metabolites in bovine lenses incubated in tissue culture medium 199 (TCM 199) with a high concentration of glucose and to evaluate the viability of this method.

Methods: We detected an accumulation of ${ }^{13} \mathrm{C}$-labeled glucose and sorbitol in the bovine lenses incubated in TCM 199 containing $50 \mathrm{mM}$ glucose- $-1{ }^{13} \mathrm{C}$ for 1,2 , 4, or 6 days using a $2 \mathrm{~T}$ CSI Omega system. ${ }^{1} \mathrm{H}^{-13} \mathrm{C}$ gradient-selected heteronuclear multiple-quantum coherence (GH-HMQC) in nuclear magnetic resonance (NMR), an indirect method of detecting nuclei in low sensitivity by ${ }^{1} \mathrm{H}$ NMR, was used to observe ${ }^{13} \mathrm{C}$-enriched metabolites in the bovine lens in vivo. Results: We found an accumulation of ${ }^{13} \mathrm{C}$-labeled glucose and sorbitol using the 2T CSI Omega system. The level of glucose increased rapidly in the posterior segment of the lens as well as in the anterior segment at one day after the beginning of incubation. Then it diminished gradually, and could no longer be detected on the 6th day after the start of incubation possibly because of the breakdown of the cell membrane. The levels of sorbitol increased in the anterior area and in the equator of the lens one day after the beginning of incubation, and remained unchanged for 6 days.

Conclusion: We encountered many problems especially with poor resolution of the imaging (consequently the time for measurement was prolonged), but this approach may be useful for non-invasive monitoring of ${ }^{13} \mathrm{C}$-labeled compounds. We believe this method will be useful for detecting various metabolites in vivo.

Nippon Ganka Gakkai Zasshi (J Jpn Ophthalmol Soc 107:571-577, 2003)

Jiro Nakamura ${ }^{1}$, Tomoko Sawada ${ }^{1,3}$, Yasuhiro Nishida ${ }^{1}$, Kazutaka Kani ${ }^{1}$, Ryohei Okamoto ${ }^{2}$, Shigehiro Morikawa ${ }^{3}$ and Toshiro Inubushi ${ }^{3}$

${ }^{1}$ Department of Ophthalmology, Shiga University of Medical Science; ${ }^{2}$ Research Center, Shiga University of Medical Science; ${ }^{3}$ Molecular Neurobiology Research Center, Shiga University of Medical Science

DOI 10.1007/s10384-004-1017-3 